\title{
Unselective screening for liver disease may not be cost effective
}

\author{
Michael Marks clinical research fellow ${ }^{1}$, Andrew Chadwick registrar ${ }^{2}$ \\ ${ }^{1}$ Clinical Research Department, London School of Hygiene and Tropical Medicine, London, UK; ${ }^{2}$ Intensive Care Medicine, Oxford University Hospitals \\ NHS Trust, Oxford, UK
}

Sattar and colleagues review the epidemiology, manifestations, and management of non-alcoholic fatty liver disease. ${ }^{1}$ They recommend considering screening for other liver conditions such as chronic viral hepatitis, autoimmune liver disease, haemochromatosis, and drug induced liver injury. The value and cost implications of such screening in people with abnormal results in liver function tests are unclear.

A prospective study in 11 primary care practices of 1290 people with abnormal liver function results established a diagnosis in less than $5 \%$ of cases. ${ }^{2}$ Only 17 (1.3\%) people were diagnosed with a condition that needed specific treatment, with most (13) having viral hepatitis.

In a study of 1118 patients in primary care, non-alcoholic fatty liver disease and alcohol related liver disease were the most common causes of abnormal liver function tests $(26.4 \%$ and $25.3 \%$, respectively). ${ }^{3}$ Autoimmune and inherited metabolic conditions accounted for only $1 \%$ each.

We reviewed clinical notes, imaging results, and test results for 338 consecutive patients with abnormal liver function results who presented to a tertiary care hospital over one year. We found that alcoholic liver disease was the most common underlying diagnosis $(22 \%)$. A thorough history and ultrasonography had the highest diagnostic yield, followed by testing for chronic viral hepatitis, which reached a diagnosis in $14 \%$ of patients. The cost per diagnosis varied substantially between tests (table $\downarrow$ ). The yield of screening for metabolic and autoimmune causes of liver disease was minimal.

Unselective diagnostic testing places a large financial burden on the NHS, ${ }^{4}$ often for limited diagnostic yield. Further prospective studies comparing different diagnostic strategies for patients with abnormal liver function tests are urgently needed to inform clinical practice.

Competing interests: None declared.

Sattar N, Forrest E, Preiss D. Non-alcoholic fatty liver disease. BMJ 2014;349:g4596. (29 July.)

2 Lilford RJ, Bentham L, Girling A, Litchfield I, Lancashire R, Armstrong D, et al. Birmingham and Lambeth Liver Evaluation Testing Strategies (BALLETS): a prospective cohort study. Health Technol Assess 2013;17:i-xiv, 1-307.

3 Armstrong MJ, Houlihan DD, Bentham L, Shaw JC, Cramb R, Olliff S, et al. Presence and severity of non-alcoholic fatty liver disease in a large prospective primary care cohort. $J$ Hepatol 2012;56:234-40.

4 Marks M. Routine test batteries for cognitive impairment in older people may not be cost effective. BMJ 2011;343:d6330.

Cite this as: BMJ 2014;349:95384

๑ BMJ Publishing Group Ltd 2014 


\section{Table}

Table 1| Diagnostic yield of investigations for abnormal liver function test results

\begin{tabular}{lcc} 
Investigation & Diagnostic yield (\%) & Cost per diagnosis (£) \\
History & 40 & N/A \\
\hline Ultrasonography & 30 & 158 \\
\hline Hepatitis A screening & 3 & 857 \\
\hline Hepatitis B screening & 4 & 1044 \\
\hline Hepatitis C screening & 7 & 265 \\
\hline Autoimmune screen & 1 & 2796 \\
\hline Metabolic tests & 0 & N/A
\end{tabular}

$£ 1=€ 1.26=\$ 1.66 . \mathrm{N} / \mathrm{A}=$ not applicable. 